\title{
Mycobacterium parascrofulaceum sp. nov., novel slowly growing, scotochromogenic clinical isolates related to Mycobacterium simiae
}

\author{
Correspondence \\ C. Y. Turenne \\ cturenne@hc-sc.gc.ca
}

\author{
C. Y. Turenne, ${ }^{1}$ V. J. Cook, ${ }^{2}$ T. V. Burdz, ${ }^{1}$ R. J. Pauls, ${ }^{3}$ L. Thibert, ${ }^{4}$ \\ J. N. Wolfe ${ }^{1}$ and A. Kabani ${ }^{1,5}$ Canada, Winnipeg, Manitoba, Canada Canada Winnipeg, Manitoba, Canada
${ }^{4}$ Laboratoire de santé publique du Québec, Institut national de santé publique du Québec, Sainte-Anne-de-Bellevue, Québec, Canada \\ ${ }^{1}$ National Reference Centre for Mycobacteriology, National Microbiology Laboratory, Health \\ ${ }^{2}$ Division of TB Control, BC Centre for Disease Control Society, Vancouver, British Columbia, \\ ${ }^{3,5}$ Faculty of Medicine ${ }^{3}$ and Department of Medical Microbiology ${ }^{5}$, University of Manitoba,
}

\section{INTRODUCTION}

Sequence-based identification of non-tuberculous mycobacteria (NTM) has become a method of choice in several mycobacteriology laboratories and is accomplished by analysis of various targets, such as the $16 \mathrm{~S}$ rRNA gene,

Published online ahead of print on 4 June 2004 as DOI 10.1099/ ijs.0.02940-0.

Abbreviations: AFB, acid-fast bacilli; BAL, bronchoalveolar lavage; CT, computed tomography; NTM, non-tuberculous mycobacteria; PRA, PCR-restriction enzyme analysis.

The GenBank/EMBL/DDBJ accession numbers for the 16S rRNA gene sequence of strain $\mathrm{HSC}-68^{\top}$ and the ITS1 and partial $h s p 65$ gene representing each sequevar are AY337273-AY337283.

Sequence identity and Hamming distances between M. parascrofulaceum and its closest established relatives, ITS1 sequence analysis and sequence alignment of sequevars $\mathrm{I}-\mathrm{V}$ are available as supplementary material in IJSEM Online.
16S-23S spacer region (ITS1) and hsp65 gene. As a result, we are faced with the discovery of many novel species previously undifferentiable using other methods of identification. These cannot be ignored, since current studies show that the detection of novel species is a common occurrence that will only be compounded with an increase in use of automated sequencers in clinical laboratories, with some of these unusual isolates deemed clinically significant (Tortoli et al., 2001). The purpose of this study was to establish as a novel species a group of representative mycobacterial strains commonly found, not only in human clinical isolates obtained from our laboratory, but also in several past publications where evidence of its existence has been found. This species has shown similarities to several known NTM species using single identification methods, which may have been the cause for the delay in its establishment, but it is clearly a unique species as determined from a polyphasic approach. In one symptomatic patient, this organism was isolated in multiple respiratory 
samples, and associated computed tomography (CT) scan and chest X-ray showed evidence of respiratory disease, as described below.

\section{METHODS}

Case history. A 41-year-old woman sought medical attention for post-landing immigration surveillance, having arrived in Canada from India in June 1999. She was asymptomatic and admitted to a history of previous pulmonary tuberculosis (TB) that was treated for 1 year in 1988 while she was in India. She denied previous BCG vaccination and $\mathrm{TB}$ skin testing. Her initial post-landing chest $\mathrm{X}$-ray showed minimal right upper lobe scarring suggestive of previous TB. Three sputum samples collected for acid-fast bacilli (AFB) smear and culture were negative. Her medical follow-up included yearly chest X-rays that remained unchanged for 2 years post-immigration.

In February 2002, her annual screening chest X-ray showed a small peripheral cavitary lesion in the right mid-lung region. She denied symptoms of TB except for a dry cough. Sputum samples were repeated for AFB smear and culture. A CT scan of the chest showed multiple calcified granulomas with scarring in the right upper lobe and calcified pre-tracheal lymph nodes. An $18 \mathrm{~mm}$ thick-walled cavity in the superior segment of the right lower lobe associated with smaller satellite nodules (the largest of which measured $12 \mathrm{~mm}$ ) was also visible. No air-fluid level was visible. Fibre-optic bronchoscopy and bronchoalveolar lavage (BAL) was performed. Samples were negative for AFB on smear. However, both sputum and bronchoscopy specimens grew an atypical mycobacterium, later identified by $16 \mathrm{~S}$ rRNA gene sequencing as Mycobacterium sp. strain MCRO 33 (described herein as Mycobacterium parascrofulaceum sp. nov.). Bronchoscopy specimens were negative for malignancy.

Repeat chest X-rays in October 2002 confirmed an enlarging cavity in the right lower lobe. Sputum samples were obtained and empirical anti-TB treatment was instituted: daily isoniazid $300 \mathrm{mg}$, rifampicin $600 \mathrm{mg}$, ethambutol $800 \mathrm{mg}$, pyrazimamide $1 \mathrm{~g}$, levofloxacin $500 \mathrm{mg}$ and vitamin $\mathrm{B}_{6} 25 \mathrm{mg}$, a regimen chosen due to concerns for atypical mycobacteria and known associated drug resistance. Her human immunodeficiency virus status was negative. After 2 months of treatment, the cavity continued to increase in size and wall thickness. Medication side-effects included oral dryness and persistent arthalgias. Liver tests remained within normal limits. Discontinuation of pyrazinamide led to resolution of her joint symptoms. The scotochromogenic mycobacterium was again isolated from her sputum and, in January 2003, the final identification and sensitivities of the organism became available. The organism was resistant to streptomycin, isoniazid, low-dose ethambutol and levofloxacin and was sensitive to rifampicin, high-dose ethambutol, clarithromycin, kanamycin, ethionamide and amikacin. On this basis, the treatment regimen was adjusted to include clarithromycin $500 \mathrm{mg}$ twice daily and high-dose ethambutol $1200 \mathrm{mg}$ and rifampicin $600 \mathrm{mg}$ once daily, a regimen that was well tolerated and which resulted in resolution of her cough.

A follow-up CT scan of the chest was performed after 3 months and showed a decrease in the thickness of the cavity wall although the overall size of the cavity had increased from 18 to $22 \mathrm{~mm}$ in maximal dimension. The dominant satellite nodule had reduced in size from $12 \mathrm{~mm}$ to less than $5 \mathrm{~mm}$. The other surrounding satellite nodules measuring less than $5 \mathrm{~mm}$ were still present and remained unchanged. Sputum cultures are now negative for $M$. parascrofulaceum.

Strain selection. Strains used in this study were obtained in three ways. Table 1 lists 12 strains obtained from patient samples. Five of these, designated 'HSC', were identified by $16 \mathrm{~S}$ rRNA gene sequencing as part of a retrospective analysis of Accuprobe-negative slowly growing NTM strains isolated at the Health Sciences Centre Mycobacteriology Laboratory, Winnipeg, Manitoba, from 1981 until 1999 (Pauls et al., 2003). These five strains had been previously identified as Mycobacterium scrofulaceum by conventional phenotypic testing and morphology. The other seven strains, designated ' $U$ ', were submitted to the National Reference Centre for Mycobacteriology for identification by other provincial public health laboratories from 2001 to 2003. Table 2 lists reference strains from the ATCC and TMC at our disposal, designated M. scrofulaceum or

Table 1. Demographics of clinical strains of Mycobacterium parascrofulaceum sp. nov.

Isolates from Manitoba were initially identified as M. scrofulaceum based on biochemical testing. Various sequevars for the 441 bp Telenti fragment of the hsp65 gene and the ITS1 spacer region are indicated for each strain. Location reflects Canadian province from which strains were isolated (BC, British Columbia). CIN, Cervical intraepithelial neoplasia.

\begin{tabular}{|lccllll|}
\hline Strain & hsp65 & ITS1 & Location & Year & \multicolumn{1}{c|}{ Source } & Comments \\
\hline HSC68 & I & I & Manitoba & 1981 & Cervix biopsy & Female, 21-year-old; diagnosed with CIN I-II \\
HSC87 & I & I & Manitoba & 1982 & Sputum & Female, 59-year-old \\
HSC90 & I & I & Manitoba & 1982 & Sputum & Female \\
HSC273 & I & I & Manitoba & 1983 & Sputum & Male, 77-year-old; squamous cell carcinoma \\
& & & & & in right main bronchus \\
HSC2054 & I & I & Manitoba & 1996 & Sputum & Female, 78-year-old \\
U01260 & IV & IV & BC & 2001 & Sputum & 64-year-old \\
U02127 & V & I & Alberta & 2002 & Sputum & Female, 70-year-old \\
U02307 & II & IIa & BC & $2002($ Feb) & Bronchial washing & Refer to case report \\
U02505 & II & IIa & & $2002(\mathrm{Oct})$ & Sputum & Refer to case report \\
U02532 & III & III & BC & 2002 & Bronchial washing & Female, 72-year-old \\
U03053 & I & I & BC & 2003 & Sputum & Male, 70-year-old \\
U03081 & II & IIb & BC & 2003 & Bronchial washing & Male, 71-year-old \\
& & & & & (right upper lobe) & \\
\hline
\end{tabular}


Table 2. Sequence-based identification of non-type reference strains initially identified as M. scrofulaceum or M. simiae

Strains were identified by sequencing of the $16 \mathrm{~S}$ rRNA gene, hsp65 and ITS1 region. Results indicate $100 \%$ sequence identity with the designated type strain of either M. scrofulaceum sensu stricto (1) or M. parascrofulaceum sp. nov. (2), unless stated otherwise. ND, Not determined.

\begin{tabular}{|lccc|}
\hline Strain & 16S rRNA & hsp65 & ITS1 \\
\hline M. scrofulaceum TMC 1302 (=ATCC 35785) & 2 & 2 & 2 \\
M. scrofulaceum ATCC 35786 & 1 & 1 & 1 (with 1 nt difference) \\
M. scrofulaceum TMC 1306 (=ATCC 35787) & 2 & 2 & 2 \\
M. scrofulaceum TMC 1307 (=ATCC 35788) & 2 & 2 & 2 \\
M. scrofulaceum TMC 1309 (=ATCC 35789) & 1 & ND & 1 (with 1 nt difference) \\
M. scrofulaceum TMC 1312 (=ATCC 35790) & 1 & ND & 1 \\
M. scrofulaceum TMC 1314 (=ATCC 35791) & 1 & 1 & 1 \\
M. scrofulaceum TMC 1315 (=ATCC 35792) & 1 & 1 & 1 \\
M. scrofulaceum TMC 1316 (=ATCC 35793) & 2 & 2 & 2 \\
M. scrofulaceum TMC 1320 (=ATCC 35794) & 2 & 2 & ND \\
M. simiae ATCC 15080 & 2 & 2 & 2 \\
\hline
\end{tabular}

Mycobacterium simiae, and deemed potential candidates for belonging to this novel species group due to phenotypic similarities. Strains M. scrofulaceum ATCC $19981^{\mathrm{T}}$ and M. simiae ATCC $25275^{\mathrm{T}}$ were also included for comparative purposes. All strains had been stored at $-80^{\circ} \mathrm{C}$ in skimmed milk with glycerol and were subcultured to Bactec12B liquid medium and Middlebrook 7H10 agar at $37^{\circ} \mathrm{C}$ for testing.

Phenotypic properties. A standard series of biochemical tests, growth temperatures and morphological examination for mycobacterium characterization were performed as described by Kent \& Kubica (1985); these characteristics are listed in Table 3.

Susceptibility testing was performed using serial twofold broth microdilution as described by Brown et al. (1992), Brown-Elliott et al. (2003), NCCLS (2003) and Wallace et al. (1986). Microdilution plates were prepared in-house using Middlebrook 7H9 broth (Difco Laboratories) supplemented with $5 \%$ oleic acid/albumin/glucose enrichment. Seven or eight concentrations were tested for each antimicrobial agent. Stock concentrations of the following antimicrobial agents were prepared from manufacturer's powders: rifampicin, ethambutol, isoniazid, amikacin and sulfamethoxazole (Sigma), ciprofloxacin and moxifloxacin (Bayer), rifabutin and linezolid (Pharmacia), streptomycin (ICN), clarithromycin (Abbott) and levofloxacin (Jansen-Othro). The isolates were tested at a final inoculum of $10^{3}-10^{4}$ c.f.u. $\mathrm{ml}^{-1}$. Microdilution plates were sealed in plastic bags and incubated at $37^{\circ} \mathrm{C}$ until macroscopic growth was sufficient for interpretation (7-14 days). Minimum inhibitory concentration (MIC) was defined as the lowest concentration of drug to inhibit visible growth with the exception of sulfamethoxazole, for which the MIC is determined at $80 \%$ inhibition of growth compared to the growth control well. Quality control was performed using Mycobacterium avium ATCC 700898, Mycobacterium marinum ATCC $927^{\mathrm{T}}$ (Wallace et al., 1986) and M. avium NJ-9141 (Heifets et al., 1993). Staphylococcus aureus ATCC 29213 was used as a quality control strain in cation-supplemented Mueller-Hinton broth when MIC ranges for mycobacteria were not available.

HPLC. Analysis of the cell wall mycolic acids was performed by HPLC with UV detection according to standard methods (Butler et al., 1996). Briefly, the mycolic acids were cleaved from the cells by saponification, extracted with chloroform and then derivatized to

Table 3. Phenotypic characteristics of M. parascrofulaceum sp. nov. clinical and reference strains ( $n=18$ unless otherwise indicated) in comparison with other slowly growing species

For the biochemical tests not listed in this table, M. avium, M. simiae and M. scrofulaceum present with the same results as for M. parascrofulaceum sp. nov. (see text). Data for reference species were taken from Goodfellow \& Magee (2001), Kent \& Kubica (1985) and Metchock et al. (1999). NG, No growth; V, variable.

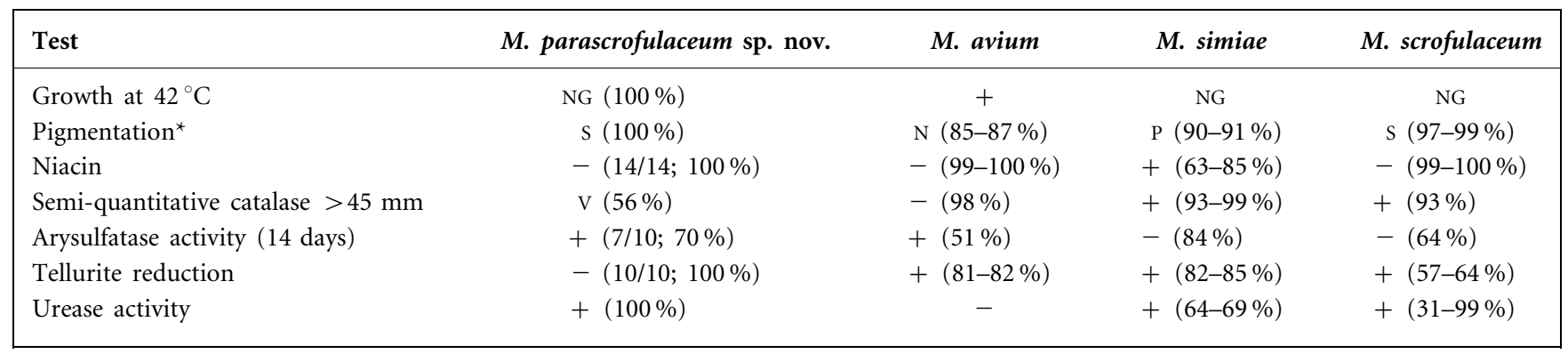

${ }^{\star} \mathrm{N}$, Non-chromogen; P, photochromogen; s, scotochromogen. 
UV-absorbing bromophenacyl esters. The esters were separated in a column, where they were eluted using a graded series of methanol and methylene chloride. For identification of the species, visual comparison of patterns with those of recognized species, in-house and published data, is still the most reliable procedure. The reference strain of Mycobacterium intracellulare ATCC $13950^{\mathrm{T}}$ was used as a control as well as a comparative strain.

Sequencing of the 16S rRNA gene and ITS1 region. A loopful of cells from pure culture was suspended in sterile distilled water, boiled for $10 \mathrm{~min}$ and then subjected to mechanical lysis using the Mini Bead-Beater (Biospec Products). The lysates were then centrifuged for $2 \mathrm{~min}$ at $12000 \mathrm{~g}$ and the supernatant was used for PCR. Amplification of the 16S rRNA gene (Rogall et al., 1990; Turenne et al., 2001) and the ITS1 region (Harmsen et al., 2002; Turenne et al., 2002) was performed as previously described and the PCR products were directly sequenced using forward and reverse primers and loaded on an ABI PRISM 310 Genetic Analyzer capillary sequencer (Applied Biosystems). Sequence editing and phylogenetic analyses by CLUSTAL V (neighbour-joining method) were performed using the SEQMAN and MEGALIGN components of LASERGENE 5 (DNASTAR).

Sequencing and PCR-restriction enzyme analysis (PRA) of the hsp65 gene. A $441 \mathrm{bp}$ fragment of the hsp65 gene was amplified from the DNA lysates prepared above using the protocol of Telenti et al. (1993). The resulting PCR product was sequenced using both the forward and the reverse PCR primers TB11 and TB12. Computer-generated PRA profiles of 441 bp sequences with enzymes HaeIII and BstEII were determined using MAPDRAW (DNASTAR) and were compared with available PRA profiles from PRASITE (http://www.hospvd.ch/prasite).

\section{RESULTS AND DISCUSSION}

\section{Phenotypic properties}

Acid-fast microscopy revealed pleomorphic forms of AFB with moderate beading. Growth was observed from 25 to $37^{\circ} \mathrm{C}$ and was optimum at $37^{\circ} \mathrm{C}$, with colonies appearing after 2-4 weeks. Dysgonic growth was observed at $25^{\circ} \mathrm{C}$, with no growth at $42^{\circ} \mathrm{C}$. On Middlebrook $7 \mathrm{H} 10$ agar medium, all strains were scotochromogenic with yellow to orange pigmentation. Colony morphology varied among strains, with most appearing smooth and domed, one appearing dry and wrinkled (HSC2054), and one showing a combination of smooth and dry (ATCC 15080). Strain U01260 was mucoid on solid media. All isolates were positive for urease, $68^{\circ} \mathrm{C}$ catalase and pyrazinamidase and negative for Tween 80 hydrolysis, nitrate reductase, iron uptake, tolerance to $5 \% \mathrm{NaCl}$, growth on MacConkey agar, niacin, $\beta$-glucosidase, acid phosphatase and 3-day arylsulfatase tests. Results were variable for the semi-quantitative catalase test and 14-day arylsulfatase test and were not attributed to any specific $h s p 65$ or ITS1 sequevars.

\section{Susceptibility testing}

According to current NCCLS guidelines for susceptibility testing of $M$. avium complex and resistance breakpoints for Mycobacterium kansasii (NCCLS, 2003), all M. parascrofulaceum isolates were sensitive to rifampicin, clarithromycin, amikacin and, with the exception of one isolate
Table 4. $\mathrm{MIC}_{50}$ and $\mathrm{MIC}_{90}$ values $\left(\mu \mathrm{g} \mathrm{ml}^{-1}\right)$ of 17 isolates of $M$. parascrofulaceum sp. nov. as determined by broth microdilution

Of the two isolates from the case study, only U02307 was included.

\begin{tabular}{|lccc|}
\hline $\begin{array}{l}\text { Antimicrobial } \\
\text { agent }\end{array}$ & $\begin{array}{c}\text { Concentration range } \\
\text { tested }\left(\boldsymbol{\mu} \mathbf{~ m l}^{-\mathbf{1}}\right)\end{array}$ & MIC $_{\mathbf{5 0}}$ & MIC $_{\mathbf{9 0}}$ \\
\hline Rifampicin & $0 \cdot 06-8 \cdot 0$ & $\leqslant 0 \cdot 06$ & $\leqslant 0 \cdot 06$ \\
Isoniazid & $0 \cdot 31-40 \cdot 0$ & $40 \cdot 0$ & $>40 \cdot 0$ \\
Streptomycin & $0 \cdot 63-80 \cdot 0$ & $2 \cdot 5$ & $20 \cdot 0$ \\
Clarithromycin & $1 \cdot 0-128 \cdot 0$ & $\leqslant 1 \cdot 0$ & $\leqslant 1 \cdot 0$ \\
Amikacin & $0 \cdot 63-80 \cdot 0$ & $5 \cdot 0$ & $10 \cdot 0$ \\
Ciprofloxacin & $0 \cdot 125-16 \cdot 0$ & $8 \cdot 0$ & $16 \cdot 0$ \\
Sulfamethoxazole & $2 \cdot 0-256 \cdot 0$ & $8 \cdot 0$ & $32 \cdot 0$ \\
Linezolid & $0 \cdot 5-64 \cdot 0$ & $\leqslant 2 \cdot 0$ & $4 \cdot 0$ \\
Moxifloxacin & $0 \cdot 03-4 \cdot 0$ & $0 \cdot 5$ & $>4 \cdot 0$ \\
& & & \\
\hline
\end{tabular}

(U02307), sulfamethoxazole (Table 4). Seventy-six per cent were susceptible to a streptomycin breakpoint of $10 \mu \mathrm{g} \mathrm{ml}^{-1}$. All isolates were resistant to isoniazid. Eighty-eight per cent of isolates were resistant to an ethambutol breakpoint of $5 \mu \mathrm{g} \mathrm{ml}^{-1}$ and $70 \%$ were resistant to the ciprofloxacin breakpoint of $2 \mu \mathrm{g} \mathrm{ml}^{-1}$. If proposed breakpoints for linezolid and slowly growing mycobacteria are followed, all isolates are susceptible to this antimicrobial (BrownElliott et al., 2003). For moxifloxacin, $71 \%$ of isolates had an MIC $\leqslant 1 \cdot 0 \mu \mathrm{g} \mathrm{ml}^{-1}$. M. scrofulaceum ATCC $19981^{\mathrm{T}}$ revealed a susceptibility pattern identical to the consensus profile for M. parascrofulaceum sp. nov. (data not shown).

\section{Mycolic acid pattern by HPLC}

The HPLC-generated pattern for isolate HSC- $68^{\mathrm{T}}$ revealed a clearly separated double cluster with prominent peaks in the early cluster. As illustrated in Fig. 1 with the reference control strain $M$. intracellulare, the pattern produced by this particular isolate of M. parascrofulaceum overlaps with and is indistinguishable from patterns seen with isolates of $M$. avium complex and some M. scrofulaceum isolates (Butler et al., 1999).

\section{Sequencing of the 16S rRNA gene}

The nearly complete $16 \mathrm{~S}$ rRNA gene sequence, from positions 28 to 1483 of the Escherichia coli sequence, was determined for strain HSC- $68^{\mathrm{T}}$ as well as the $5^{\prime}$ region, from positions 54 to 510 , of all subsequent isolates. All isolates were identical to strain $\mathrm{HSC}-68^{\mathrm{T}}$ in the $5^{\prime}$ region. Strains that showed some variation in either the ITS1 or hsp65 sequence from the type strain were subsequently fully sequenced for the $16 \mathrm{~S}$ rRNA gene, although no other mismatches from HSC- $68^{\mathrm{T}}$ were present. The sequence of M. parascrofulaceum contains the characteristic $12 \mathrm{bp}$ omission (short helix) in the hypervariable region 18 (V3) observed in rapidly growing mycobacteria, M. simiae and other slowly growing species related to M. simiae, but which 

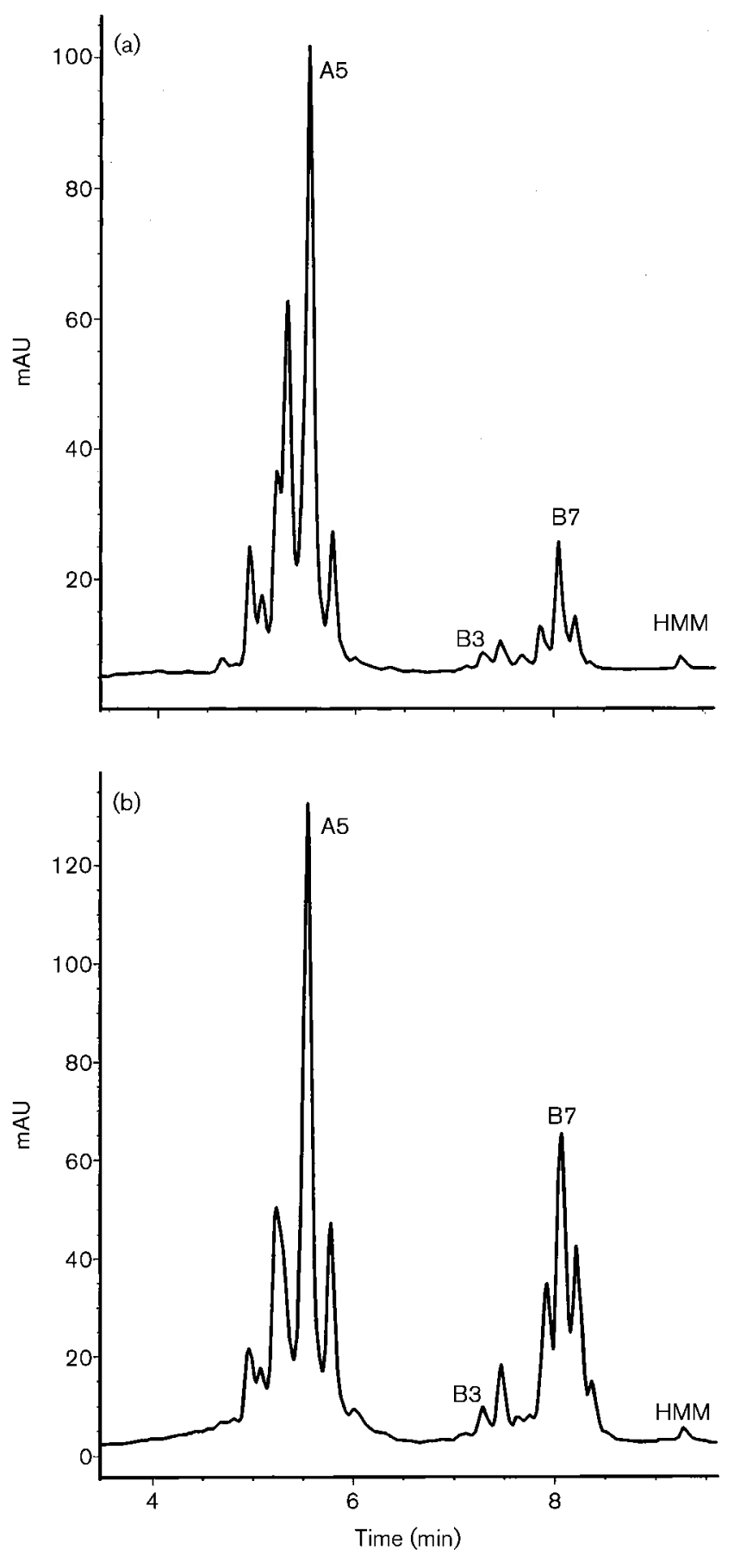

Fig. 1. Mycolic acid patterns of $M$. parascrofulaceum sp. nov. strain $\mathrm{HSC68}^{\top}$ (a) and M. intracellulare ATCC $13950^{\top}$ (b) obtained by HPLC analysis. Peak designation is as previously suggested (Glickman et al., 1994). HMM, High molecular mass internal standard.

is not found in M. scrofulaceum. Analysis of the nearly complete 16S rRNA gene sequence using BLAST against sequences from the GenBank, EMBL or DDBJ databases revealed an identical match with Mycobacterium sp. MCRO 33 (AF152559), followed by Mycobacterium sp. MCRO 19
(X93030) with $6 \mathrm{nt}$ differences, and Mycobacterium palustre BAA $-377^{\mathrm{T}}$ (AJ308603) and Mycobacterium sp. MCRO 19 (X93030) both with $14 \mathrm{nt}$ differences. The closest match using the RIDOM database (Harmsen et al., 2002), from E. coli bp 54-510, was [M. simiae] ATCC 15080, with $100 \%$ identity. This strain is not the type strain of $M$. simiae and the RIDOM results prompted us to include this strain among the misidentified reference strains suspected of belonging to $M$. parascrofulaceum sp. nov. The second closest match by RIDOM was M. simiae DSM $44165^{\mathrm{T}}$ with seven mismatches. Complete $16 \mathrm{~S}$ rRNA gene sequence comparisons with $M$. simiae revealed a 19 bp mismatch ( $98 \cdot 6 \%$ identity).

Final sequence analysis was done using our in-house sequence database (in combination with GenBank sequences) and evaluating from bases 54 to 1470 on the E. coli $16 \mathrm{~S}$ rRNA gene (Fig. 2). Sequence identity and Hamming distances against the closest established species are available as Supplementary Table A in IJSEM Online. An alignment of hypervariable regions $\mathrm{A}$ and $\mathrm{B}$ reveals signature sequences in the A region (Fig. 3), with the $\mathrm{B}$ region being identical to that of $M$. simiae.

\section{Sequencing of the ITS1}

The ITS1 sequences determined for all strains identified as $M$. parascrofulaceum on the basis of $16 \mathrm{~S}$ rRNA gene sequence analysis revealed five sequevars, designated sqv. I, IIa, IIb, III and IV. Sequevars I, IIa, IIb and III differ from each other by 1-2 bases only (Supplementary Fig. A in IJSEM Online). Sequevar IV is the most distant from sqv. I by $13 \mathrm{bp}$. Neither of the sequences corresponded to ITS1 sequences in public databases or RIDOM.

BLAST search analysis of sqv. I revealed closest similarity with various sequences designated M. scrofulaceum. Highest scores were for M. scrofulaceum KPM 2027 (AB026702) and M. scrofulaceum ATCC $19981^{\mathrm{T}}\left(=\mathrm{TMC} 1323^{\mathrm{T}}\right)(\mathrm{L} 15622)$, both with five mismatches over $\sim 350$ bases, and identical to M. scrofulaceum ATCC $19981^{\mathrm{T}}$ determined in-house. Shorter GenBank entries (280 bases) resulting in a lower score were in fact $100 \%$ identical to sqv. I: M. scrofulaceum C58 (AJ314885) and M. scrofulaceum ATCC 19073 (AJ314884). As these are not representative of the type strain, we assume that these also belong to $M$. parascrofulaceum. RIDOM analysis results indicated $100 \%$ identity over 280 bases with [M. simiae] ATCC 15080, followed by M. scrofulaceum S343 (four mismatches) and M. scrofulaceum DSM $43992^{\mathrm{T}}$ (five mismatches) and finally sequences of $M$. intracellulare, with $28 \mathrm{nt}$ differences and above.

Analysis of sqv. IV revealed a closest match with $M$. scrofulaceum DSM $43992^{\mathrm{T}}$ (11 mismatches) using RIDOM and the same M. scrofulaceum sequences as determined for sqv. I using BLAST, but with 12-15 mismatches from entries corresponding to the type strain of M. scrofulaceum or 6-7 mismatches from entries corresponding to sqv. I. 


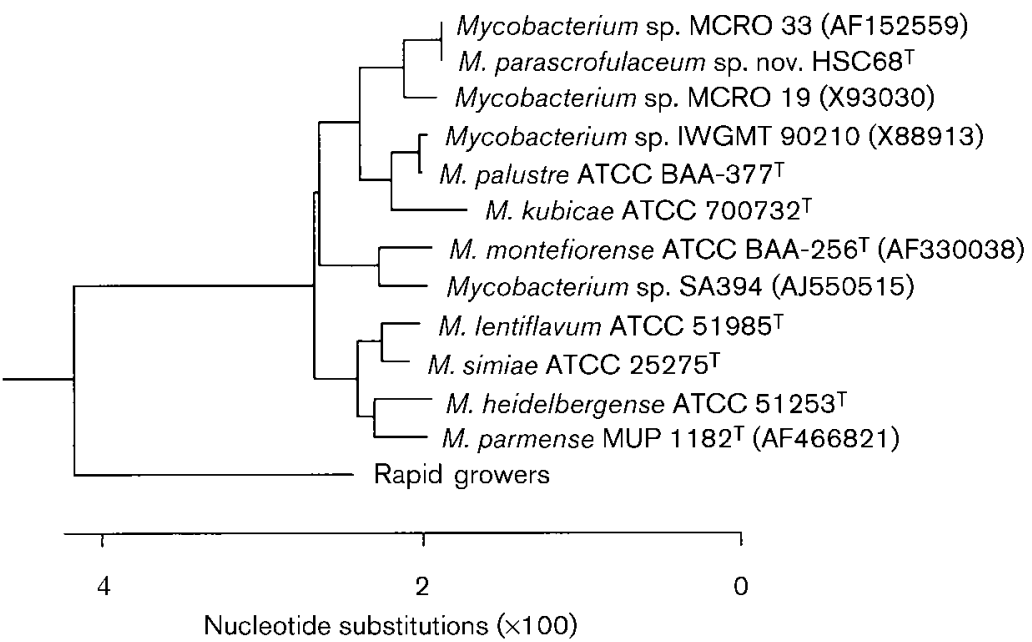

Fig. 2. Phylogenetic tree of Mycobacterium parascrofulaceum sp. nov. with its closest genotypic and phenotypic relatives. CLUSTAL $W$ analysis was performed using the MEGALIGN component of the LASERGENE software (DNASTAR). End gaps were removed to include positions 54 to 1470 of the E. coli 16S rRNA gene. Nocardia asteroides served as the outgroup sequence. Sequences were determined in our laboratory unless indicated by a GenBank accession number in parentheses.

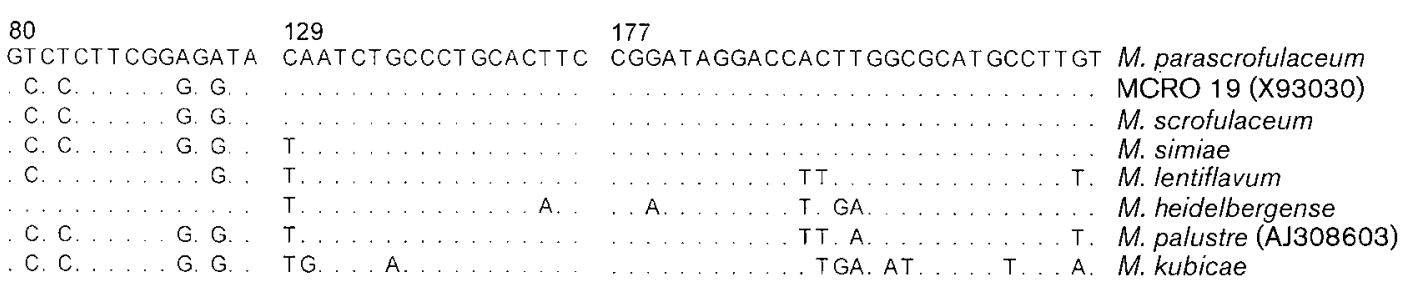

Fig. 3. Alignment of hypervariable region $A(V 2)$ of the $16 \mathrm{~S}$ rRNA gene. Nucleotide positions indicated are according to the E. coli sequence. With the exception of one base difference in strain MCRO 19, all strains listed in the alignment have an identical hypervariable region B sequence and this is therefore not included. Species are represented by the corresponding type strain. Sequences were determined in our laboratory unless indicated by a GenBank accession number.

\section{Sequencing and PRA of the hsp65 gene}

Sequences of the $h s p 65441 \mathrm{bp}$ region of strains of M. parascrofulaceum revealed five distinct sequevars, sqv. I-V (Table 5; Supplementary Fig. B), with one or two base variations from each other, with the exception of strains U02532 (sqv. II) and U02127 (sqv. V), differing by 17 and 23 bases from sqv. I, respectively. Sequevar I was the most prevalent, followed by sqv. II, which differed from sqv. I by one base at position 129 of the 441 bp fragment. Sequevar IV, represented by strain U01260 alone, differed from sqv. I by two bases at positions 189 and 327 of the $441 \mathrm{bp}$ fragment. Sequevars I, II and IV are most closely related to M. scrofulaceum ATCC $19981^{\mathrm{T}}$, which differs from sqv. I by 16 bases. The partial protein sequences of the $441 \mathrm{bp}$ fragments reveal two groups, the first consisting of sqv. I, II and IV and the other consisting of sqv. III and V. They differ from each other by three amino acids.

Table 5. Computer-generated PRA patterns using hsp65 gene sequences of the various M. parascrofulaceum sequevars and its closest relatives by HPLC, $16 \mathrm{~S}$ rRNA, ITS 1 and biochemical analysis

Results are given as fragment sizes in bp; fragments below $50 \mathrm{bp}$ are omitted. The score obtained from PRASITE reflects the distance between the result and query, i.e. the smaller the distance, the greater the resemblance. In general, computer-generated PRA data will differ slightly from RFLP data, giving a score of approximately 10 for known identical strains (data not shown). Results above 10 generally suggest a different pattern.

\begin{tabular}{|lccl|}
\hline hsp65 sequevar & BstEII & HaeIII & Best results using PRASITE (score) \\
\hline I (12 strains) & $231 / 116 / 79$ & $139 / 94$ & 1. M. lentiflavum type 4: 240/130/85-130/95 (20) \\
II (3 strains) & $231 / 116 / 79$ & $127 / 94$ & 2. M. lentiflavum type 4: 240/130/85-130/95 (18) \\
III (1 strain) & $231 / 210$ & $127 / 94 / 81 / 78$ & 3. M. kansasii type 1: 240/210-130/105/80 (15) \\
IV (1 strain) & $231 / 131 / 79$ & $127 / 94$ & 4. M. lentiflavum type 4: 240/130/85-130/95 (11) (probable match) \\
V (1 strain) & $231 / 210$ & $145 / 139 / 75$ & 5. M. scrofulaceum type 1: 240/210-145/130/95 (24) \\
\hline
\end{tabular}


BLAST analysis of the partial $h s p 65$ sequence was performed with sqv. I, III and V, using from bp 46 to 405 of the $441 \mathrm{bp}$ fragment to obtain the most accurate highest score with mycobacterial $h s p 65$ sequences in the public database, most being either 401 or $360 \mathrm{bp}$ in length. BLAST results for sqv. 1 revealed a highest score with [Mycobacterium gordonae] SN 601 (AF434735) of $100 \%$ identity; this is believed to be misidentified, as it shows $30 \mathrm{nt}$ differences from the type strain, M. gordonae ATCC $14470^{\mathrm{T}}$. The next closest matches were M. scrofulaceum (AF547871 and AF434733), with $96 \%$ similarity (16 bp variation), corresponding to that determined with our in-house sequence of the type strain. BLAST results of strain U02532 (sqv. III) revealed a highest score and 12 mismatches (in $360 \mathrm{bp)}$ with M. avium complex strain 121B-2 (AF241211), which differs from the type strain of $M$. avium by $16 \mathrm{bp}$, followed by [M. gordonae] SN 601 (AF434735), also with 16 bp variations. BLAST results with strain U02127 (sqv. V) revealed a highest score with Mycobacterium bohemicum CIP $105811^{\mathrm{T}}$ (AF547811), $95 \%$ similarity (15 mismatches), followed by both Mycobacterium interjectum DSM $44064^{\mathrm{T}}$ (AF547846) and $M$. avium complex strain 121B-2 (AF241211), with $94 \%$ similarity or $19 \mathrm{nt}$ differences.

Computerized restriction maps of the $441 \mathrm{bp} h s p 65$ fragment as determined by MAPDRAW (LaserGene) using enzymes BstEII and HaeIII revealed a unique pattern for each sequevar, with the exception of sqv. IV, whose pattern is close to that of Mycobacterium lentiflavum type 4 as determined by PRASITE. These patterns are listed in Table 5; fragments smaller than $50 \mathrm{bp}$ were not taken into account.

M. scrofulaceum was first described by Prissick \& Masson (1956) and was often grouped with M. avium, M. intracellulare and related organisms to form the so-called MAIS complex. It has long since been established as a distinct species, with its main characteristics being orange or yellow pigmentation, positive reaction for urease, negative for Tween, semi-quantitative catalase $>45 \mathrm{~mm}$ and negative nitrate results. M. scrofulaceum is found in the environment and is an opportunistic pathogen associated with childhood lymphadenitis, disseminated infections, pulmonary disease and skin infection (Aikin \& Johnston, 1978; Corbett et al., 1999; Dustin et al., 1980; Hsueh et al., 1996; Joshi et al., 1989; Murray-Leisure et al., 1987; Prissick \& Masson, 1956; Sanders et al., 1995; Wolinsky, 1979, 1995). However, in most of these clinical cases, identification of the organism was obtained via conventional methods, such as pigmentation and biochemical testing. A recent increase in novel mycobacterial species due to molecular-based techniques, including scotochromogenic slowly growing species, suggests that these species probably always existed in clinical samples but were unidentifiable by conventional methodology. This idea was built upon by Tortoli (2003), who stated a possible link between a decrease in cases of M. scrofulaceum lymphadenitis and the recent discovery of several novel species of scotochromogenic slowly growing mycobacterial species.
In our study, most isolates of $M$. parascrofulaceum were obtained from respiratory samples. Determining the role of the organism in the disease process was difficult owing to a lack of medical records. Medical charts were reviewed for two of the Winnipeg isolates, but the isolates could not indisputably be associated with disease (Pauls et al., 2003). However, the clinical case described here represents a clear example of the pathogenic potential of this organism.

\section{Similar species reported in other studies}

We found that a great proportion of reference strains from ATCC and TMC, and also clinical isolates identified as $M$. scrofulaceum prior to sequence-based testing, were deemed to belong to the novel species $M$. parascrofulaceum. This is not surprising given the phenotypic similarities between the two species. However, molecular data, such as from sequencing of the 16S rRNA gene, hsp65 and ITS1, clearly distinguish this species from $M$. scrofulaceum. Springer et al. (1996) first documented this organism (which they designated MCRO 33) and described it as a unique slowly growing species related to $M$. simiae based on 16S rRNA gene analysis but most closely resembling $M$. avium-M. intracellulare-M. scrofulaceum (or MAIS complex) based on biochemical testing. Since then, other laboratories that use sequence-based methods for identification of mycobacterial species have shown the presence of this previously unidentified species in clinical samples, either by recognition of a $16 \mathrm{~S}$ rRNA gene sequence similarity with MCRO 33 or through our own analyses of their sequences (published or deposited in GenBank).

A BLAST analysis using only the first 500 bases of the $16 \mathrm{~S}$ rRNA gene revealed additional GenBank submissions with identical sequences to that of 'MCRO 33'. These consisted of unspeciated strains Mycobacterium sp. N228 (AY215339) (Hall et al., 2003) and Mycobacterium sp. N625 (AY215248) (Hall et al., 2003), assumed to be derived from clinical specimens. Although using only the partial gene for analysis is satisfactory for clinical strains in a routine laboratory, it is important to bear in mind that analysis using the nearly complete $16 \mathrm{~S}$ rRNA gene remains the best strategy for accurate eubacterial speciation and is essential for taxonomic studies.

Holberg-Petersen et al. (1999) identified a clinical isolate (strain M.16/89) as M. scrofulaceum by conventional testing, but with a clearly distinct $16 \mathrm{~S}$ rRNA gene sequence closer to M. simiae and a lipid profile of MAIS. They suggested the strain may not belong to either M. simiae or M. scrofulaceum (Holberg-Petersen et al., 1999). Our evaluation of their 16S rRNA gene hypervariable regions $\mathrm{A}$ and $\mathrm{B}$ indicated perfect identity with $M$. parascrofulaceum. Tortoli et al. (2001), using a polyphasic approach to decipher the taxonomic status of unusual mycobacteria, described two strains (FI-12100 and FI-25197) identified by conventional testing as M. lentiflavum, by HPLC as MAC and by $16 \mathrm{~S}$ rRNA gene sequencing as MCRO 33. Strain FI-25197 was 
obtained from blood of an AIDS patient and was therefore deemed of probable clinical significance.

Swanson et al. (1996) recognized five isolates of $M$. scrofulaceum as having a distinct hsp65 gene sequence (corresponding to our sqv. 1) along with a 16S rRNA gene sequence corresponding to MCRO 33, and suggested that this group probably represented a novel species. One of these strains, Lane 3081, was also part of a past study that identified this strain as having the 'MAC-G' ITS1 sequevar (corresponding to our ITS1 sqv. I) (Frothingham \& Wilson, 1993) and has been described as 'exhibiting cultural, biochemical and serological properties characteristic of M. scrofulaceum' (Saito et al., 1990; Tomioka et al., 1993). Four of five clinical isolates in Brazil identified as M. scrofulaceum by da Silva Rocha et al. (2002) revealed a 'new' pattern similar to our sqv. II: $235 / 115 / 85-125 / 95$, as determined by gel electrophoresis.

Roth et al. (2000), using the ITS1 spacer for mycobacterial identification, revealed five taxonomically uncertain strains closest to M. scrofulaceum. Based on a partial ITS1 alignment of these strains, we determined that one corresponds to our ITS1 sqv. III and the other four to our sqv. IIb. Roth et al. (2000) also determined that these strains had a distinctive $16 \mathrm{~S}$ rRNA gene sequence that was identical to that of MCRO 33. Because many researchers have already detected this organism in past studies and with sequence-based methods being commonplace in laboratories, it seems inevitable that this organism be described as a distinct species. We propose the name Mycobacterium parascrofulaceum sp. nov. for this unique Mycobacterium species.

\section{Description of Mycobacterium parascrofulaceum sp. nov.}

Mycobacterium parascrofulaceum (pa.ra.scro.fu.la'ce.um. Gr. prep. para like; N.L. neut. adj. scrofulaceum specific epithet of a bacterial species; N.L. neut. adj. parascrofulaceum referring to the phenotypic resemblance of the isolate to M. scrofulaceum).

Microscopy shows pleomorphic bacillary forms of AFB with moderate beading. Strains are slowly growing and scotochromogenic and grow on Löwenstein-Jensen medium at $25-37^{\circ} \mathrm{C}$, optimally at $37^{\circ} \mathrm{C}$. Isolates are positive for urease, $68^{\circ} \mathrm{C}$ catalase and pyrazinamidase; negative for Tween 80 hydrolysis, nitrate reductase, iron uptake, tolerance to $5 \%$ $\mathrm{NaCl}$, growth on MacConkey agar, niacin, $\beta$-glucosidase, acid phosphatase and 3-day arylsulfatase; variable results seen for the semi-quantitative catalase test and 14-day arylsulfatase test. Morphology and biochemical profile indistinguishable from $M$. scrofulaceum. Isolates show in vitro susceptibility to rifampicin, clarithromycin, amikacin and sulfamethoxazole and resistance to isoniazid. The organism is almost identical to MAC and some M. scrofulaceum by HPLC. Various molecular targets confirm this organism as a distinct species. 16S rRNA gene sequence analysis reveals that the organism contains the characteristic short helix 18 of the M. simiae group and whose closest established species is the recently described M. palustre. Five sequevars each of hsp65 and ITS1 have been observed among isolates of $M$. parascrofulaceum, none corresponding to established strains of known species in public sequence databases.

The type strain, HSC $-68^{\mathrm{T}}$ (=ATCC BAA- $614^{\mathrm{T}}=\mathrm{DSM}$ $44648^{\mathrm{T}}$ ), was the first isolate studied in our laboratory and was representative of the most common $h s p 65$ or ITS1 sequevar (I) of M. parascrofulaceum. Most isolates were from human respiratory samples and, in at least one case, was felt to be clinically significant, requiring antimycobacterial therapy.

\section{REFERENCES}

Aikin, K. R. \& Johnston, R. F. (1978). Tuberculous-like disease produced by M. scrofulaceum. Pa Med 81, 38-41.

Brown, B. A., Wallace, R. J., Jr \& Onyi, G. O. (1992). Activities of clarithromycin against eight slowly growing species of nontuberculous mycobacteria, determined by using a broth microdilution MIC system. Antimicrob Agents Chemother 36, 1987-1990.

Brown-Elliott, B. A., Crist, C. J., Mann, L. B., Wilson, R. W. \& Wallace, J. R., Jr (2003). In vitro activity of linezolid against slowly growing nontuberculous mycobacteria. Antimicrob Agents Chemother 47, 1736-1738.

Butler, W. R., Floyd, M. M., Silcox, V. A. \& 9 other authors (1996). Standardized method for HPLC identification of mycobacteria. Atlanta, GA: Centers for Disease Control and Prevention, US Department of Health and Human Services.

Butler, W. R., Floyd, M. M., Silcox, V. A. \& 9 other authors (1999). Mycolic acid pattern standard for HPLC identification of mycobacteria. Atlanta, GA: Centers for Disease Control and Prevention, US Department of Health and Human Services.

Corbett, E. L., Hay, M., Churchyard, G. J., Herselman, P., Clayton, T., Williams, B. G., Hayes, R., Mulder, D. \& De-Cock, K. M. (1999). Mycobacterium kansasii and M. scrofulaceum isolates from HIVnegative South African gold miners: incidence, clinical significance and radiology. Int $J$ Tuberc Lung Dis 3, 501-507.

da Silva, R. A., Werneck Barreto, A. M., Dias Campos, C. E., Villas-Boas, D. S., Fonseca, L., Saad, M. H., Degrave, W. M. \& Suffys, P. N. (2002). Novel allelic variants of mycobacteria isolated in Brazil as determined by PCR-restriction enzyme analysis of hsp65. J Clin Microbiol 40, 4191-4196.

Dustin, P., Demol, P., Derks-Jacobovitz, D., Cremer, N. \& Vis, H. (1980). Generalized fatal chronic infection by Mycobacterium scrofulaceum with severe amyloidosis in a child. Pathol Res Pract 168, 237-248.

Frothingham, R. \& Wilson, K. H. (1993). Sequence-based differentiation of strains in the Mycobacterium avium complex. J Bacteriol 175, 2818-2825.

Glickman, S. E., Kilburn, J. O., Butler, W. R. \& Ramos, L. S. (1994). Rapid identification of mycolic acid patterns of mycobacteria by high-performance liquid chromatography using pattern recognition software and a Mycobacterium library. J Clin Microbiol 32, 740-745.

Goodfellow, M. \& Magee, J. G. (2001). Taxonomy of mycobacteria. In Mycobacteria. I: Basic Aspects, pp. 1-71. Edited by P. R. Gangadharam \& P. A. Jenkin. New York: International Thomson Publishing. 
Hall, L., Doerr, K. A., Wohlfiel, S. L. \& Roberts, G. D. (2003). Evaluation of the MicroSeq system for identification of mycobacteria by $16 \mathrm{~S}$ ribosomal DNA sequencing and its integration into a routine clinical mycobacteriology laboratory. J Clin Microbiol 41, 1447-1453. Harmsen, D., Rothganger, J., Frosch, M. \& Albert, J. (2002). RIDOM: ribosomal differentiation of medical micro-organisms database. Nucleic Acids Res 30, 416-417.

Heifets, L., Lindholm-Levy, P., Libonati, J. P., Hooper, N., Laszlo, A., Cynamon, M. \& Siddiqi, S. (1993). Radiometric broth macrodilution method for the determination of minimal inhibitory concentrations (MIC) with Mycobacterium avium complex isolates. Denver, CO: National Jewish Center for Immunology and Respiratory Medicine. Holberg-Petersen, M., Steinbakk, M., Figenschau, K. J., Jantzen, E., Eng, J. \& Melby, K. K. (1999). Identification of clinical isolates of Mycobacterium spp. by sequence analysis of the $16 \mathrm{~S}$ ribosomal RNA gene. Experience from a clinical laboratory. APMIS 107, 231-239.

Hsueh, P. R., Hsiue, T. R., Jarn, J. J., Ho, S. W. \& Hsieh, W. C. (1996). Disseminated infection due to Mycobacterium scrofulaceum in an immunocompetent host. Clin Infect Dis 22, 159-161.

Joshi, W., Davidson, P. M., Jones, P. G., Campbell, P. E. \& Roberton, D. M. (1989). Non-tuberculous mycobacterial lymphadenitis in children. Eur J Pediatr 148, 751-754.

Kent, P. T. \& Kubica, G. P. (1985). Public Health Mycobacteriology: a Guide for the Level III Laboratory. Atlanta, GA: Department of Health and Human Services, Centers for Disease Control.

Metchock, B., Nolte, F. S. \& Wallace, R. J., Jr (1999). Mycobacterium. In Manual of Clinical Microbiology, pp. 399-437. Edited by P. R. Murray, E. J. Baron, M. A. Pfaller, F. C. Tenover \& R. H. Yolken. Washington, DC: American Society for Microbiology.

Murray-Leisure, K. A., Egan, N. \& Weitekamp, M. R. (1987). Skin lesions caused by Mycobacterium scrofulaceum. Arch Dermatol 123, 369-370.

NCCLS (2003). Susceptibility testing of mycobacteria, nocardiae, and other aerobic actinomycetes. Approved Standard M24-A. Wayne, PA: National Committee for Clinical Laboratory Standards.

Pauls, R. J., Turenne, C. Y., Wolfe, J. N. \& Kabani, A. (2003). A high proportion of novel mycobacteria species identified by $16 \mathrm{~S}$ rDNA analysis among slowly growing Accuprobe negative strains in a clinical setting. Am J Clin Pathol 120, 560-566.

Prissick, F. H. \& Masson, A. M. (1956). Cervical lymphadenitis in children caused by chromogenic mycobacteria. J Can Med Assoc 75, 798-803.

Rogall, T., Wolters, J., Flohr, T. \& Bottger, E. C. (1990). Towards a phylogeny and definition of species at the molecular level within the genus Mycobacterium. Int J Syst Bacteriol 40, 323-330.

Roth, A., Reischl, U., Streubel, A., Naumann, L., Kroppenstedt, R. M., Habicht, M., Fischer, M. \& Mauch, H. (2000). Novel diagnostic algorithm for identification of mycobacteria using genus-specific amplification of the 16S-23S rRNA gene spacer and restriction endonucleases. J Clin Microbiol 38, 1094-1104.

Saito, H., Tomioka, H., Sato, K., Tasaka, H. \& Dawson, D. J. (1990). Identification of various serovar strains of Mycobacterium avium complex by using DNA probes specific for Mycobacterium avium and Mycobacterium intracellulare. J Clin Microbiol 28, 1694-1697.

Sanders, J. W., Walsh, A. D., Snider, R. L. \& Sahn, E. E. (1995). Disseminated Mycobacterium scrofulaceum infection: a potentially treatable complication of AIDS. Clin Infect Dis 20, 549-556.

Springer, B., Stockman, L., Teschner, K., Roberts, G. D. \& Bottger, E. C. (1996). Two-laboratory collaborative study on identification of mycobacteria: molecular versus phenotypic methods. J Clin Microbiol 34, 296-303.

Swanson, D. S., Pan, X. \& Musser, J. M. (1996). Identification and subspecific differentiation of Mycobacterium scrofulaceum by automated sequencing of a region of the gene (hsp65) encoding a 65kilodalton heat shock protein. J Clin Microbiol 34, 3151-3159.

Telenti, A., Marchesi, F., Balz, M., Bally, F., Bottger, E. C. \& Bodmer, T. (1993). Rapid identification of mycobacteria to the species level by polymerase chain reaction and restriction enzyme analysis. J Clin Microbiol 31, 175-178.

Tomioka, H., Saito, H., Sato, K., Tasaka, H. \& Dawson, D. J. (1993). Identification of Mycobacterium avium complex strains belonging to serovars $21-28$ by three commercial DNA probe tests. Tuber Lung Dis 74, 91-95.

Tortoli, E. (2003). Impact of genotypic studies on mycobacterial taxonomy: the new mycobacteria of the 1990s. Clin Microbiol Rev 16, 319-354.

Tortoli, E., Bartoloni, A., Bottger, E. C. \& 8 other authors (2001). Burden of unidentifiable mycobacteria in a reference laboratory. J Clin Microbiol 39, 4058-4065.

Turenne, C. Y., Tschetter, L., Wolfe, J. \& Kabani, A. (2001). Necessity of quality-controlled 16S rRNA gene sequence databases: identifying nontuberculous Mycobacterium species. J Clin Microbiol 39, 3637-3648.

Turenne, C., Chedore, P., Wolfe, J., Jamieson, F., Broukhanski, G., May, K. \& Kabani, A. (2002). Mycobacterium lacus sp. nov., a novel slowly growing, non-chromogenic clinical isolate. Int J Syst Evol Microbiol 52, 2135-2140.

Wallace, R. J., Jr, Nash, D. R., Steele, L. C. \& Steingrube, V. (1986). Susceptibility testing of slowly growing mycobacteria by a microdilution MIC method with 7H9 broth. J Clin Microbiol 24, 976-981.

Wolinsky, E. (1979). Nontuberculous mycobacteria and associated diseases. Am Rev Respir Dis 119, 107-159.

Wolinsky, E. (1995). Mycobacterial lymphadenitis in children: a prospective study of 105 nontuberculous cases with long-term follow-up. Clin Infect Dis 20, 954-963. 